\title{
CRONIUS RUBER (LAMARCK, 1818), UN NUEVO DECÁPODO EN LOS FONDOS SOMEROS DE CANARIAS
}

\author{
Yolanda Maggio*, Jorge Núñez, José Ramón Docoito*, \\ Leopoldo Moro $^{* *}$, Rogelio Herrera ${ }^{* *}$, José Carlos Hernández
}

\section{RESUMEN}

Estudio observacional sobre la llegada y el estado actual de las poblaciones del cangrejo decápodo pantropical Cronius ruber (Lamarck, 1818) en la isla de Tenerife. Se trata de una especie de la familia Portunidae, cuyos primeros registros publicados en Canarias se producen en el año 2017, siendo citada por primera vez en el litoral de Tenerife en el ańo 2018; aunque por la información recabada para este trabajo, los primeros registros de la presencia de esta especie en Tenerife se remontan al ańo 2008. Se trata de una especie neocolonizadora a tener en cuenta por su potencialidad invasora en los archipiélagos de Madeira y Canarias. Su aparición en las Islas ha sido relacionada principalmente con el intenso transporte marítimo entre Canarias y África occidental; sin embargo, no podemos descartar una expansión natural de sus poblaciones relacionada con el calentamiento reciente de las aguas de Canarias, propicias para el establecimiento de especies tropicales. Con este estudio pretendemos además definir el nicho ecológico ocupado por $C$. ruber y valorar de manera cualitativa el grado de competencia con otras especies de crustáceos decápodos nativos presentes en el litoral de Canarias.

Palabras Clave: especie neocolonizadora, cambio climático, transporte marítimo, Portunido, hábitats costeros, islas.

CRONIUS RUBER (LAMARCK, 1818), A NEW DECAPOD

IN THE SHALLOW BOTTOMS OF THE CANARIES

\section{Abstract}

Observational study about the arrival and the actual status of the populations of the pantropical decapod crab Cronius ruber (Lamarck, 1818) in the island of Tenerife. It is a species of the family Portunidae, whose first records in the Canary Islands were published in 2017, being cited for the first time on the coast of Tenerife in 2018. Although due to the information collected in this work, the first records of the presence of this species in Tenerife date back to 2008. It is a neocolonizer specie and a very important one because of its potential to act as an invasive species in the archipelagos of Madeira and the Canary Islands. Its arrival to the islands have been related with the intense maritime transports between the Canaries and occidental Africa. However, we cannot be ruled out a natural expansion of its populations related with the recent water warming of the Canaries, favorable to the establishment of tropical species. With this study, we aim to define the ecological niche occupied by $C$. ruber and to qualitative assess the degree of competition with other species of native decapods crustaceans present on the Canary littoral.

Keywords: neocolonizer species, climate change, marine transport, Portunid, costal habitats, Islands. 


\section{INTRODUCCIÓN}

Recientemente, la especie de cangrejo Cronius ruber (Lamarck, 1818) ha llegado a Canarias y en base a las observaciones de pescadores, buceadores aficionados y científicos su número está en aumento. Los primeros registros publicados de Cronius ruber en Canarias fueron en el año 2017 para la isla de Gran Canaria (González et al., 2017; Triay-Portella et al., 2018). Sin embargo, ya existen avistamientos en todo el archipiélago (https://redpromar.org/app/guide/species/cronius-ruber). Lo que pone de manifiesto la clara tendencia expansiva de esta especie en Canarias.

Los portúnidos en sentido amplio o cangrejos nadadores (superfamilia Portunoidea Rafinesque, 1815) pertenece al subphylum Crustacea y orden Decapoda; se trata de un grupo de cangrejos bien representados en los ecosistemas marinos (De Grave et al., 2009), siendo también importante el registro fósil. Su distribución es amplia, apareciendo en todos los océanos y mares del mundo, desde las zonas costeras litorales hasta grandes profundidades. Muchas de las especies de portúnidos forman parte de niveles tróficos altos, ya que se definen como depredadores estrictos. Son especies que ocupan hábitats blandos de gravas, arenas y limos, jugando un papel trófico clave en estos ambientes (Abelló, 1989; Koch y Duriš, 2016).

En general, algunas de las especies de Portunoidea, debido a su tamaño, sus densidades poblacionales y su carne fina, son consumidos como un apreciado marisco en diversas áreas geográficas, siendo de gran importancia algunas especies en la economía de la región (Holthuis, 1987). Podemos citar a la comunidad de Galicia, donde el consumo local de nécoras (Necora puber (Linnaeus, 1767), Fam. Polybiidae Ortmann, 1893) y su exportación son de gran importancia en la economía de esa comunidad desde hace mucho tiempo. Un caso más reciente se ha producido en el delta del Ebro, donde el portúnido Callinectes sapidus Rathbun, 1896, conocido por su nombre vulgar «jaiba azul», invade todo el delta del Ebro, produciéndose una rápida expansión por zonas similares en todo el mar Mediterráneo. Aunque los cambios en estos ecosistemas son evidentes, por el asentamiento masivo de estas poblaciones, con alta tasa reproductiva, también se ha propuesto para su posible utilización como marisco, debido a la rápida reproducción junto con sus propiedades culinarias, y el alto valor nutricional de este portúnido, llegando a su comercialización en lonja.

En la actualidad, en Canarias se han citado unas 25 especies de Portunoidea (González-Pérez, 2018). Debido a la ecología y etología de los Portunoidea, pues se trata de cangrejos con gran movilidad y capacidad de natación, su capacidad de

* Departamento de Biología Animal, Edafología y Geología, Universidad de La Laguna, Tenerife. Canary Islands, Spain. Autor para la correspondencia: José Carlos Hernández (jocarher@) ull.es).

** Consejería de Educación. Universidades, Cultura y Deportes.

*** Servicio de Biodiversidad, Gobierno de Canarias, Edif. Usos Múltiples I, Av. Anaga n. ${ }^{\circ}$ 35, Pl. 11, 38071 S/C de Tenerife, Canary Islands, Spain. 
neocolonización es alta; además, en las últimas décadas esta capacidad se ha visto potenciada por las actividades humanas y el incremento descontrolado del tráfico marítimo (Koch y Ďuriš, 2016).

Tenerife y Gran Canaria, donde se realizaron las primeras observaciones de la especie en cuestión, son las islas con mayor tráfico portuario de Canarias. En los últimos años hay que destacar también que se ha incrementado la llegada de plataformas petrolíferas de zonas tropicales (Atlántico Nororiental) (Falcón 2015), las cuales traen consigo gran número de organismos, incluso trasladando ecosistemas enteros, que potencialmente pueden adaptarse a vivir en Canarias. También se registró la presencia de varias especies de cnidarios escleractínidos de los géneros Tubastraea y Oculina, las cuales tienen una distribución básicamente tropical, siendo estas especies también introducidas a través del puerto de Las Palmas y, como vector de transmisión, el tránsito marítimo de plataformas petrolíferas (Brito et al., 2017); lo mismo que ha ocurrido con varias especies de peces litorales (Falcón, 2015).

Por lo señalado anteriormente, el tráfico marítimo podría ser el modo de llegada de esta especie a las Islas, tal y como sugieren González et al. (2017). Sin embargo, la lejanía a los puertos comerciales de los primeros registros de esta especie en Canarias, que se aportan en este trabajo, apoya la hipótesis de que su llegada haya ocurrido de manera natural mediante larvas. El calentamiento actual de las aguas que rodean a Canarias (Hernández, 2016), además de la cercanía a los trópicos, hacen de Canarias una zona subtropical idónea para la expansión septentrional de muchas especies tropicales. Así pues, quedan importantes cuestiones por resolver que hacen muy necesaria la toma de datos que nos ayuden a dibujar fielmente la vía de llegada de esta especie, así como a determinar el rol que va a jugar en los ecosistemas marinos de Canarias.

El objetivo principal de este trabajo es sacar a la luz datos no publicados hasta el momento que adelantan el año de aparición de esta especie en Canarias y que sirven además para evaluar la evolución de sus poblaciones en el litoral de Tenerife. Por otro lado, y de manera complementaria, basándonos en nuestras observaciones definimos el nicho ecológico que está ocupando esta especie en comparación con el resto de fauna decápoda nativa. Por último, articulamos una serie de recomendaciones para paliar su posible efecto invasor.

\section{MATERIAL Y MÉTODOS}

La metodología empleada ha consistido básicamente en la realización de muestreos directos, para la observación y visualización pasiva de ejemplares de Cronius ruber, en diferentes estaciones del litoral de Tenerife, que aparecen pormenorizados en la tabla 1, con las distintas localidades en donde se procedió al muestreo, en diferentes columnas se señalan el tipo de muestreo (diurno/nocturno) y la apreciación cualitativa del grado de presencia de $C$. ruber en cada estación, siendo el resultado «nulo» cuando no se visualizaron ejemplares durante el tiempo de muestreo. Los muestreos han sido desarrollados en diferentes meses, a partir del año 2008 hasta la actualidad, por biólogos marinos expertos y conocedores de la fauna de crustáceos 


\begin{tabular}{|c|c|c|c|c|c|c|c|c|}
\hline \multicolumn{9}{|c|}{$\begin{array}{l}\text { TABLA 1. ESTACIONES DE MUESTREO DE CRONIUS RUBER } \\
\text { Y DATOS RELATIVOS A CADA OBSERVACIÓN }\end{array}$} \\
\hline 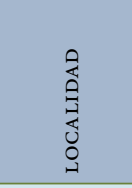 & 疋 & 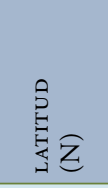 & $\begin{array}{l}0 \\
5 \\
0 \\
z \\
0 \\
0\end{array}$ & 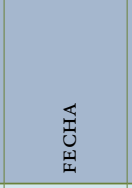 & 壳 & 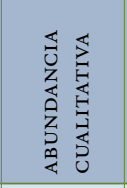 & 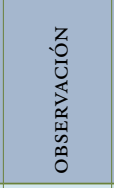 & 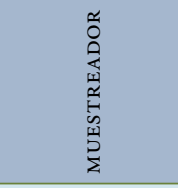 \\
\hline Teño & Pta. Diente de Ajo & 28.3420 & -16.9172 & 29-jun-08 & diurna & escaso & & J.R. Docoito \\
\hline Teño & $\begin{array}{l}\text { Cabezo de la } \\
\text { Jábiga }\end{array}$ & 28.3270 & -16.8981 & 5-jul-08 & diurna & escaso & & J.R. Docoito \\
\hline $\begin{array}{l}\text { Los } \\
\text { Gigantes }\end{array}$ & $\begin{array}{l}\text { Pta. de los } \\
\text { Infiernillos }\end{array}$ & 28.2900 & -16.8663 & 17 -ago-08 & diurna & escaso & & J.R. Docoito \\
\hline Güímar & El Puertito & 28.2939 & -16.3749 & 22-may-11 & $\begin{array}{c}\text { diurnal } \\
\text { nocturna }\end{array}$ & escaso & & J.R. Docoito \\
\hline Armeñime & Puertito de Adeje & 28.1124 & -16.7683 & $1-\operatorname{mar}-09$ & $\begin{array}{c}\text { diurnal } \\
\text { nocturna }\end{array}$ & abundante & & J.R. Docoito \\
\hline Armeñime & Puertito de Adeje & 28.1124 & -16.7683 & Ene-dic12 & $\begin{array}{c}\text { diurna/ } \\
\text { nocturna }\end{array}$ & abundante & & J.R. Docoito \\
\hline Armeñime & El Balito & 28.1409 & -16.7897 & $1-$ jul-16 & diurna & abundante & & J.C. Hernández \\
\hline $\begin{array}{l}\text { Playa } \\
\text { San Juan }\end{array}$ & $\begin{array}{l}\text { Playa de Agua } \\
\text { Dulce }\end{array}$ & 28.1034 & -16.4835 & 1-jul-17 & diurna & abundante & & J.C. Hernández \\
\hline Las Eras & Playa y saliente & 28.1929 & -16.4223 & $1-\mathrm{may}-17$ & diurna & escaso & & J.C. Hernández \\
\hline Armeñime & Puertito de Adeje & 28.1124 & -16.7683 & 1 -oct-17 & $\begin{array}{c}\text { diurnal } \\
\text { nocturna }\end{array}$ & abundante & & J.R. Docoito \\
\hline $\begin{array}{l}\text { Montańa } \\
\text { Amarilla }\end{array}$ & Caletilla & 28.0094 & -16.6384 & 1-ago-17 & diurna & abundante & $\begin{array}{l}\text { pareja en } \\
\text { cópula }\end{array}$ & J.C. Hernández \\
\hline Teño & Pta. Diente de Ajo & 28.3420 & -16.9172 & 1-may-18 & diurna & escaso & & J.C. Hernández \\
\hline Armeñime & $\begin{array}{l}\text { Puertito } \\
\text { de Adeje }\end{array}$ & 28.1124 & -16.7683 & 1-jun-19 & $\begin{array}{c}\text { diurnal } \\
\text { nocturna }\end{array}$ & abundante & & J.R. Docoito \\
\hline $\begin{array}{l}\text { Los } \\
\text { Gigantes }\end{array}$ & $\begin{array}{l}\text { Pta. de las } \\
\text { Higueras }\end{array}$ & 28.2829 & -16.8636 & 21-ago-19 & diurna & escaso & & J.R. Docoito \\
\hline $\begin{array}{l}\text { Los } \\
\text { Gigantes }\end{array}$ & $\begin{array}{l}\text { Ensenada de } \\
\text { la Frescura }\end{array}$ & 28.2738 & -16.8572 & 22-ago-19 & diurna & escaso & & J.R. Docoito \\
\hline Armeñime & Puertito de Adeje & 28.1124 & -16.7683 & 1-sep-19 & diurna & abundante & & J.R. Docoito \\
\hline Armeñime & Puertito de Adeje & 28.1124 & -16.7683 & 30-ago-19 & $\begin{array}{c}\text { diurna } \\
\text { /nocturna }\end{array}$ & escaso & & J. Núńez \\
\hline El Médano & Pta. del Médano & 28.0447 & -16.5323 & 30-sep-19 & diurna & escaso & & J. Núñez \\
\hline El Médano & Pta. del Médano & 28.0447 & -16.5323 & 16 -oct-19 & diurna & escaso & $\begin{array}{l}\text { hembra } \\
\text { ovígera }\end{array}$ & J.C. Hernández \\
\hline $\begin{array}{l}\text { Porís de } \\
\text { Abona }\end{array}$ & Playa del Muelle & 28.1653 & -16.4314 & 14-sep-19 & diurna & escaso & & J. Núńez \\
\hline Armeñime & Puertito de Adeje & 28.1124 & -16.7683 & 1 -oct-19 & diurna & escaso & $\begin{array}{l}\text { pareja en } \\
\text { cópula }\end{array}$ & J. Núńez \\
\hline Güímar & $\begin{array}{l}\text { Playa del Cabezo- } \\
\text { sector B, C }\end{array}$ & 28.2893 & -16.3775 & Ene-dic-19 & diurna & nulo & & J. Núńez \\
\hline $\begin{array}{l}\text { Icod de } \\
\text { los Vinos }\end{array}$ & San Marcos & 28.3764 & -16.7240 & $27-f e b-20$ & diurna & escaso & & J.R. Docoito \\
\hline
\end{tabular}




\begin{tabular}{|c|c|c|c|c|c|c|c|c|}
\hline Güímar & $\begin{array}{l}\text { Playa del Cabezo- } \\
\text { sector B, C }\end{array}$ & 28.2893 & -16.3775 & $\begin{array}{l}\text { Ene- } \\
\text { may-20 }\end{array}$ & diurna & nulo & & J. Núñez \\
\hline Güímar & $\begin{array}{l}\text { Playa del Náutico } \\
\text { a los Túneles }\end{array}$ & 28.2848 & -16.3821 & 1-may-20 & diurna & nulo & & J. Núñez \\
\hline Güímar & $\begin{array}{l}\text { Playa del Cabezo- } \\
\text { sector B, C }\end{array}$ & 28.2893 & -16.3775 & 24-jul-20 & diurna & nulo & & J. Núñez \\
\hline Adeje & La Caleta & 28.0990 & -16.7567 & 1-ago-20 & diurna & $\begin{array}{c}\text { muy } \\
\text { abundante }\end{array}$ & $\begin{array}{l}\text { hembra } \\
\text { ovígera }\end{array}$ & J.C. Hernández \\
\hline Teño & Pta. Diente de Ajo & 28.3420 & -16.9172 & 1 -ago-20 & diurna & $\begin{array}{c}\text { muy } \\
\text { abundante }\end{array}$ & & J.C. Hernández \\
\hline $\begin{array}{l}\text { Boca } \\
\text { Cangrejo }\end{array}$ & $\begin{array}{l}\text { Playa cerca } \\
\text { escollera } \\
\text { La Nea }\end{array}$ & 28.4056 & -16.3158 & 1-ago-20 & diurna & escaso & & J.C. Hernández \\
\hline Abades & Pta. de Abades & 28.1409 & -16.4364 & 1-ago-20 & diurna & escaso & & J.C. Hernández \\
\hline
\end{tabular}

decápodos de Canarias. Cada muestreo ha consistido en realizar sesiones de media hora de buceo con equipo ligero, abarcando una franja ancha de unos 2,5 metros en las zonas en donde posiblemente podrían encontrarse ejemplares de $C$. ruber. El muestreo pasivo consiste en observar la presencia de ejemplares sin manipulación del sustrato, es decir, sin volteo de bolos o la utilización de cualquier producto agresivo para el medio. En una tablilla de metacrilato se iban apuntando el número de ejemplares sin determinación del sexo de los mismos, aunque en algún caso se encontraron ejemplares en cópula. Solo en dos localidades, en El Médano y en el Puertito de Armeñime, se llegaron a colectar ejemplares a mano, con la protección de guantes.

El material colectado ha consistido en dos ejemplares (macho y hembra) que estaban copulando descaradamente en el Puertito de Armeñime (fig. 1). En El Médano se colectó una sola hembra con incubación de óvulos, la cual se mantuvo un tiempo en los acuarios del laboratorio produciéndose el desove de larvas zoeas de manera espontánea.

Para la caracterización del hábitat se utilizó una cámara de vídeo GoPro 7.

\section{RESULTADOS}

SisTEMÁTICA Y DESCRIPCIÓN

Filo ARTROPODA

Subfilo CRUSTACEA

Clase MALACOSTRACA

Orden DECAPODA

Familia PORTUNIDAE (Rafinesque, 1815)

La superfamilia Portunoidea incluye a las especies conocidas vulgarmente como «cangrejos nadadores», caracterizados por presentar un caparazón aplanado, 


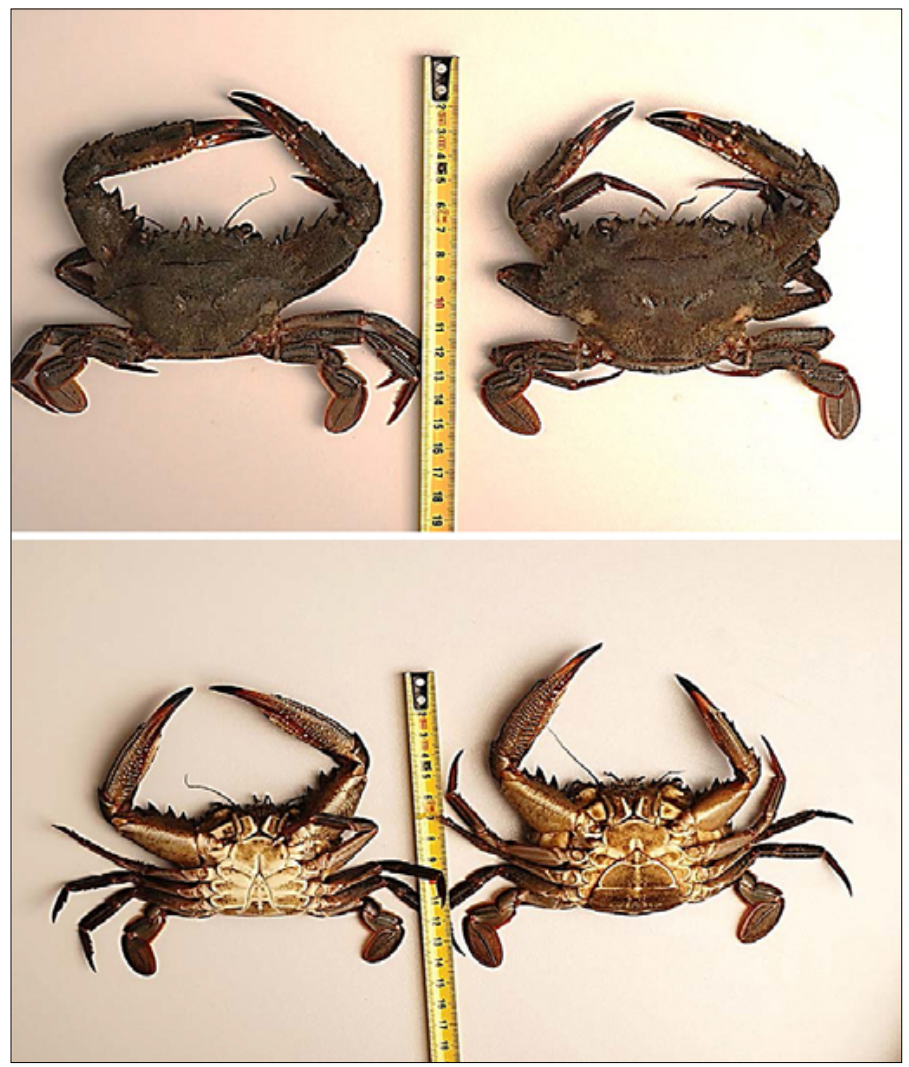

Figura 1. Ejemplares de macho (izquierda) y hembra (derecha) de Cronius ruber recolectados mientras copulaban en el Puertito de Armeñime.

en la mayoría más ancho que largo, con una serie de 5-9 dientes anterolaterales. La zona frontal más o menos horizontal o ligeramente arqueada, con las órbitas y pedúnculos oculares moderadamente largos y con una serie de espinas laterales bien desarrolladas. Una de las características más comunes de estos cangrejos es la de poseer el último par de patas adaptado tanto para la natación como para enterrarse en los sustratos blandos, para lo cual poseen el dactilopodio transformado en una paleta, aplanada y de forma más o menos ovalada (fig. 3).

En la actualidad, las diferentes subfamilias que que englobaba la familia Portunidae han sido elevadas a nivel de familia (Brusiniidae Števčić, 1991; Carcinidae MacLeay, 1838; Geryonidae Colosi, 1923; Ovalipidae Spiridonov, Neretina \& Schepetov, 2014; Polybiidae Ortmann, 1893; Portunidae Rafinesque, 1815; y Thiidae Dana, 1852) y agrupadas en la superfamilia Portunoidea, siendo Cronius Stimpson, 1860 un género monoespecífico de la subfamilia Thalamitinae Paulson, 1875 (fam. Portunidae). 

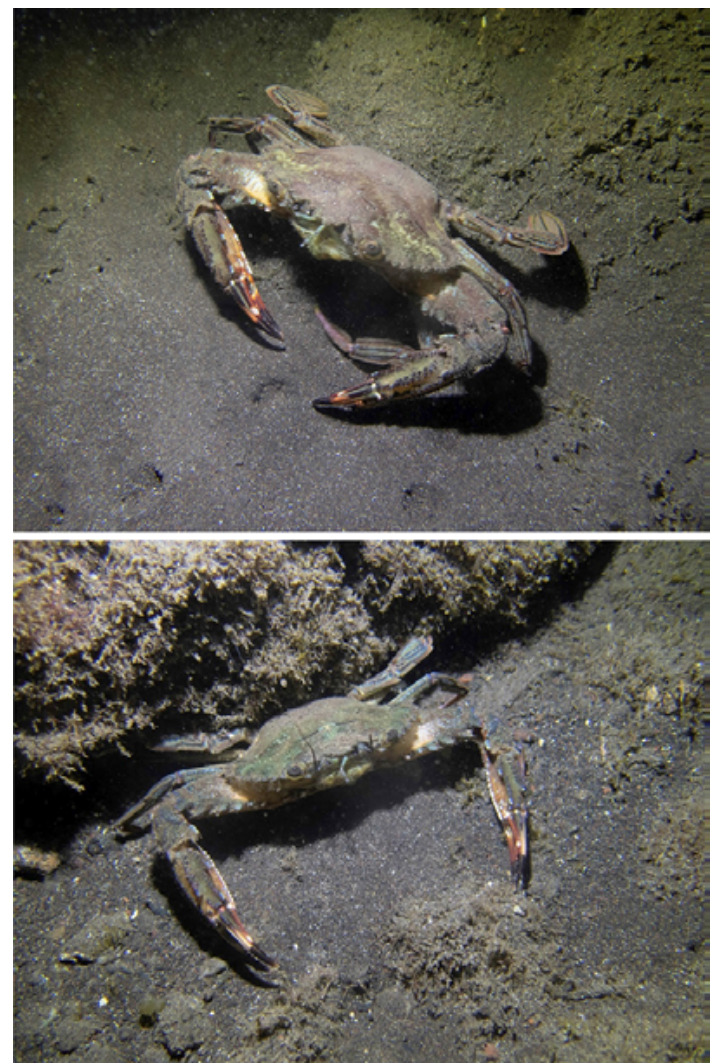

Figura 2. Ejemplares de Cronius ruber en su hábitat natural, al borde de su refugio. Interfase roca-arena.

\section{Diagnosis de CRONIUS RUBER}

Medidas del caparazón.- Los machos adultos tienen unos $50 \mathrm{~mm}$ de largo por $75 \mathrm{~mm}$ de ancho; las hembras suelen tener mayor tamaño que los machos, con una anchura de unos $85 \mathrm{~mm}$ y $55 \mathrm{~mm}$ de largo (fig. 1). Estas medidas, tomadas con los ejemplares de Tenerife, coinciden con las de otras latitudes.

Coloración.- Rojo violeta o rojo púrpura intenso, más o menos veteado con tonos más claro o blanquecinos; la parte distal de todas las espinas de color negro (Rathbun, 1930). Los ejemplares observados en Tenerife muestran una coloración más críptica, con una pubescencia pardo-verdosa (fig. 2), muy parecida a la nécora de Galicia.

Descripción morfológica (fig. 3 como apoyo para la descripción del plan corporal).Caparazón hexagonal, liso, pubescente; una cresta transversal sinuosa que se extiende entre las espinas laterales y otra cresta biarcuada más corta, trans- 
versal, a medio camino entre esta cresta y la parte frontal. Zona frontal con cuatro espinas, sin incluir las orbitales internas; un par de espinas submedianas más prominentes; el segundo par son más puntiagudas, y dirigidas ligeramente hacia atrás, estando separadas de los orbitales internos por muescas de corte más o menos profundas (fig. 1). La órbita es casi de forma circular. El artejo basal de las antenas presenta una espina debajo de la inserción de la porción móvil (articulada). Las espinas anterolaterales son desiguales, se presentan alternando grandes y pequeñas; la última espina lateral no se encuentra notablemente desarrollada como en otras especies de portúnidos. Los quelípedos son fuertes y alargados; el mero lleva de 4-6 espinas hacia la parte externa (frontal), con una pequeña espina distal en la parte de atrás; carpo con crestas granuladas, una espina interna grande y tres espinas pequeñas en la superficie externa; dáctilo con crestas granuladas en toda la superficie, armada con cuatro espinas en la zona superior, dos en el borde interior y dos en el exterior (según Williams, 1965).

Reproducción.- Datos de zonas del Atlántico occidental, se registran hembras ovígeras desde mayo a septiembre en Curazao, en junio y en julio en el litoral de Cuba y Jamaica respectivamente (Rathbun, 1930). Durante los muestreos realizados en Tenerife se ha encontrado solo una hembra ovígera en octubre, que coincide también con la época de reproducción de otros decápodos como Maja brachydactyla (centollo) en Canarias. No obstante, analizando los reportes registrados en la RedPROMAR se ha constatado la presencia en Canarias de hembras ovígeras desde finales de julio hasta finales de noviembre, mientras que las cópulas se han registrado de entre los meses de febrero y agosto, aunque con observaciones puntuales en noviembre (R. Herrera, com. pers.). Este amplio periodo con actividad reproductora puede ser una de las causas por las que su expansión haya sido tan rápida, pero son necesarios más muestreos dirigidos a conocer cuándo son los picos reproductivos en Canarias.

Hábitat y distribución.- El hábitat preferente de esta especie es en zonas sublitorales someras $(1-20 \mathrm{~m})$ y pedregosas con diversas facies algales y con interfases arenosas. De hábitos nocturnos, siendo definida como una especie esciáfila, aunque también es posible encontrarla, pero en menos densidad poblacional, en horas diurnas.

Su distribución es pantropical: costa del Atlántico occidental desde Carolina del Norte hasta Florida, golfo de Méjico, América Central, las Antillas, norte de Sudamérica desde las Guayanas a Brasil (de Amapá a Rio Grande del Sur), costas del Atlántico oriental desde Senegal hasta Angola, Macaronesia (islas de Cabo Verde, Madeira y Canarias), costa del Pacífico oriental desde California hasta Perú e Islas Galápagos (Fransozo et al., 2002).

En la última década su distribución se ha visto ampliada, ya que se hacen registros por primera vez en Canarias, con anterioridad ya era conocido de las islas de Cabo Verde y Senegal (González et al., 2017). Recientemente, también ha sido registrado en el litoral de Madeira, lo que representa los registros más septentriona- 

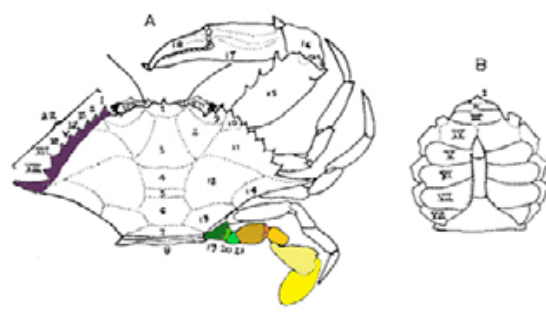

1. Frente

12. Región mesobranquial

2. Región protogástrica

3. Región mesogístrica

4. Región metagástrica

5. Región urogistrica

6. Región cardiaca

7. Región intestinal

8. Región abdominal

9. Región orbital

10. Región hepítica

11. Región epibranquial
13. Regiớn metabranquial

14. Región posterolateral

15. Merus

16. Cerpus

17. Propodus

18. Dactylus

19. Coxa

20. Basis

21. Izquium

22. Dientes enterocolaterales

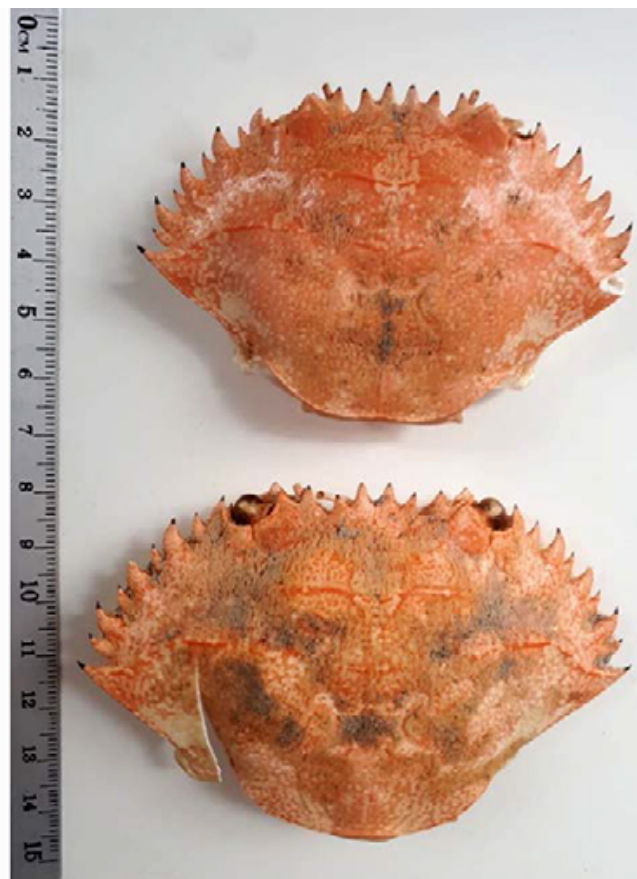

Figura 3. Izquierda: A. Portúnido en visión dorsal. B. Esternón torácico y placa abdominal en visión ventral. Derecha: Caparazón de un macho (arriba) y una hembra (debajo) de Cronius ruber.

les y un paso más hacia el norte en su distribución en el Atlántico oriental (Schäfer et al., 2019).

\section{Sobre el hábitat de CRONIUS RUBER}

Si atendemos a la Directiva Hábitats (D. 92/43/EEC), la definición de hábitat marino es «área acuática diferenciada por sus características geográficas, abióticas y bióticas, ya sean enteramente naturales o seminaturales, en las cuales viven las especies en cualquier estado del ciclo de su vida». Se trata de una definición muy general por la que, de acuerdo con Frasschetti et al. (2008), el hábitat constituye el medio físico en donde viven los organismos, teniendo en cuenta que muchos de los organismos sésiles forman parte constituyente de ese medio físico, ya que estas poblaciones se instalan sobre el sustrato primario, siendo soporte para otras especies, aumentando la complejidad del conjunto del hábitat. En esta sección definimos de manera muy general algunos de los hábitats donde se encuentra Cronius ruber en la isla de Tenerife. 
Los ejemplares que nosotros hemos observado o estudiado provienen de la isla de Tenerife, sobre todo de las costas este, sur y oeste. La mayoría de las observaciones han sido sin colecta, solo de manera puntual se han colectado ejemplares, en concreto tres ejemplares colectados a mano, dos en Armeñime (Adeje) y uno en El Médano, en total dos hembras y un macho.

En general, los fondos donde se han localizado son bastante irregulares, de tipo mixtos, con dominancia de fondos de cantos y pedregales con interfases arenosas, limoso-arenosas, en profundidades someras entre 1,5 y $6 \mathrm{~m}$ (fig. 4), que están de acuerdo con las características del hábitat y rango batimétrico definido para esta especie. En algunas zonas de la costa oeste, los muestreos se realizaron en las proximidades de cuevas submarinas, sobre todo en la entrada de las mismas, en donde los fondos son también rocosos, con cornisas, y existen pequeñas interfases de arena y limo, y a veces grava y picón. En ninguno de los muestreos realizados se llegó a zonas de praderas, ni de caulerpales ni sebadales, aunque sí existen visualizaciones de esta especie en estos hábitats de Canarias (González et al., 2017).

Para comprobar si la especie es de hábitos crepusculares y nocturnos, también se realizaron algunos muestreos de visualización nocturna, observándose un patrón similar a otros portúnidos como por ejemplo la nécora (Necora puber Linnaeus, 1767) en aguas de Galicia, cuya actividad nocturna es su comportamiento más habitual. Durante las horas diurnas suelen aparecer activos, aunque en bajo número de ejemplares y, generalmente, se encuentran refugiados en fisuras y grietas, con capacidad para semienterrarse en las interfases arenosas y limosas, con un comportamiento agresivo de defensa, enseñando las quelas, y de movimientos muy rápidos, similar a una nécora de Galicia. Durante los muestreos, en solo una ocasión se observó cópula en periodo diurno y también se llegó a colectar una hembra ovígera, también en muestreo diurno, entre septiembre y octubre, que coincide en Canarias también con la reproducción del centollo canario (Maja brachydactyla Balss, 1922). Aunque no se realizaron muestreos específicos para la determinación correcta de su dieta, su comportamiento alimentario es similar al de la nécora gallega, mostrándose como una especie omnívora que consume invertebrados y pequeños brotes algales con detritus. En cautividad se ha comprobado la potencia de sus quelas, siendo capaz de trocear pequeños erizos. Todas estas observaciones, ya corroboradas por otros equipos de investigadores, la definen como una especie ya establecida en el litoral de Canarias, e indican que $C$. ruber ya se ha instaurado en ecosistemas sublitorales canarios y con actividad reproductiva (González et al., 2017).

El portúnido $C$. ruber se caracteriza como una especie euritérmica y eurihalina, que habita en variados tipos de fondo (Le Loeuff et al., 1968); en áreas de África occidental se ha constatado que se trata de una especie de fondos someros y que habita en gran variedad de sustratos desde rocosos a sustratos blandos de tipo grava, arena y limo (Manning \& Holthuis, 1981, Fransen, 1991). En otras áreas geográficas se ha registrado desde el intermareal, incluso en charcos de marea hasta profundidades próximas a los 30 m (Manning \& Holthuis, 1981; Fransen, 1991), aunque existen algunos registros hasta $70 \mathrm{~m}$.

El tipo de fondo en donde se ha instalado C. ruber en Tenerife lo definimos como un sustrato rocoso de naturaleza volcánica situado inmediatamente por debajo 

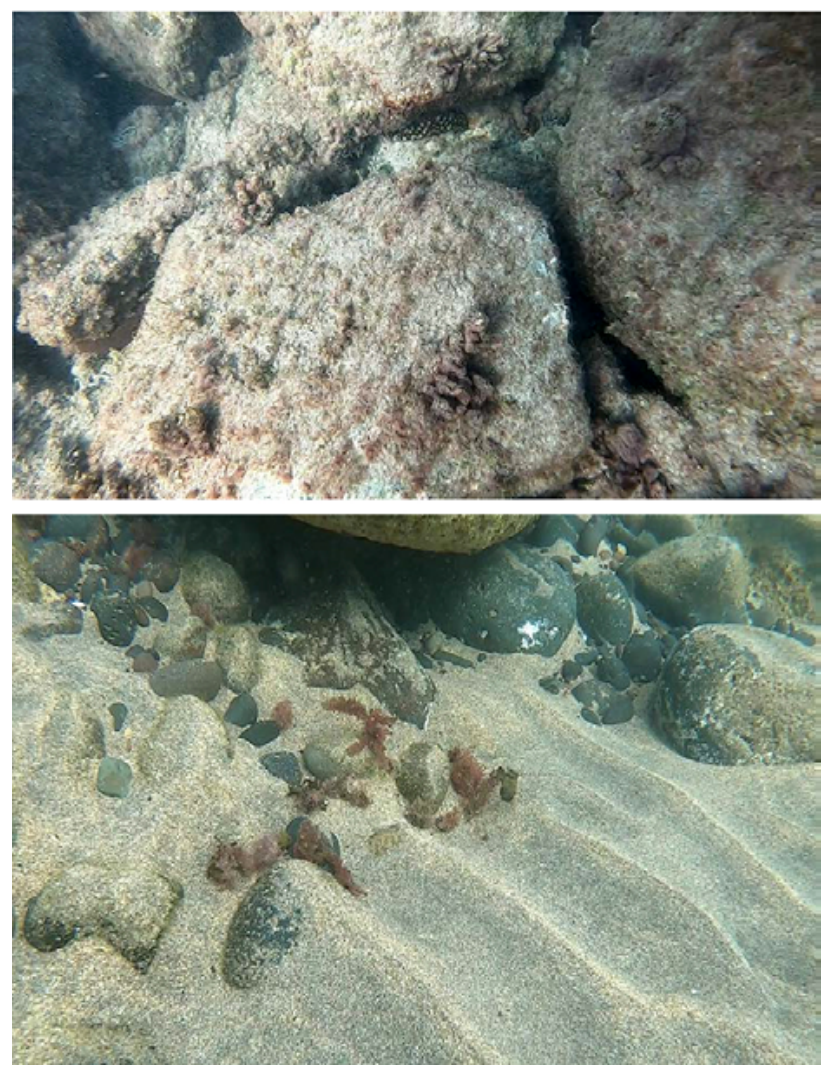

Figura 4. Ejemplos de hábitats predregosos con algas cespitosas con interfases arenosas.

del nivel de la bajamar y permanentemente sumergido (sublitoral). Las comunidades que se establecen, en general, son muy diversas, ya que se encuentran distintas facies con diferentes algas dominantes, en función de la iluminación, la exposición al oleaje, la inclinación y el tipo de sustrato, incluso se trata de facies de algas estacionales que cambian a lo largo del año. Aunque todas las facies no están constituidas por comunidades de macrófitos, en nuestro caso, la proximidad de poblaciones del erizo Arbacia lixula ha sido evidente, con zonas menos colonizadas por algas, con excepción de coralináceas incrustantes, especialmente en fondos de callao de zonas expuestas; mientras que en las áreas resguardadas es sustituido por otro erizo, Paracentrotus lividus, la holoturia Holothuria sactori y la ofiura Ophioderma longicaudum. A medida que progresamos en profundidad, llegamos a zonas de blanquizales en donde es dominante el erizo de púas largas Diademum africanum, también presente en los hábitats de C. ruber.

Las comunidades de cuevas y tubos volcánicos submarinos, sobre todo en los tramos de entrada, son un refugio para muchas especies móviles, que encuentran 
en estos enclaves su hábitat idóneo, habiéndose observado ejemplares de $C$. ruber en algunos enclaves de la isla de Tenerife. En estas áreas las comunidades de macrófitos algales son escasas y las especies presentes son generalmente algas esciáfilas calcáreas en la entrada de las cuevas y tubos, siendo frecuentes los géneros Peyssonnelia y Lithothamnium.

\section{DISCUSIÓN}

Se confirma la expansión de las poblaciones del decápodo portúnido Cronius ruber en el litoral somero de la isla de Tenerife, sobre todo en zonas de caletas abrigadas. Las poblaciones muestran su estado ovígero en los meses más cálidos, entre julio y octubre, coincidente con otros crustáceos de hábitos similares como el centollo (Maja brachydactyla), y solapándose en parte con otras áreas más cálidas del Atlántico caribeño en donde aparecen hembras ovígeras entre mayo y septiembre. No se han estudiado cambios en los ecosistemas donde habita, ocupando un nicho ecológico del submareal somero que en principio no estaba ocupado por otro decápodo similar. En estas zonas del litoral pulpos y sepias (chocos) pueden actuar como depredadores del C. ruber, según constatamos al entrevistar a mariscadores y submarinistas. Desde el punto de vista de su control, se trata de una especie de fácil recolección, ya sea a mano o con la utilización de nasas específicas para su captura, pudiendo ser controladas sus poblaciones; incluso tiene el potencial de convertirse en un recurso marisquero nuevo en las Islas.

Ya hemos comentado que los decápodos portúnidos, en general, son especies con gran movilidad, buenos nadadores, gregarios y con gran capacidad de colonización, ya que muchas de las especies presentan una amplia distribución de tipo pantropical. Por otra parte, la tasa reproductiva es alta, ya que está constatado que $C$. ruber es una especie que se aparea durante todo el año, lo que, unido a su régimen alimentario de gran espectro, la hace capaz de adaptarse a cualquier tipo de fondo somero. Estos son factores que contribuyen a que la especie se comporte como neocolonizadora de forma natural y que pueda tener un carácter de especie invasora en su nuevo hábitat, alterando la estructura de las comunidades en las que está presente.

En las aguas de Canarias $C$. ruber se ha registrado desde hace más de una década, en concreto en la isla de Tenerife, aunque como ya hemos comentado anteriormente, el dato se presenta ahora en esta publicación. Es evidente que en la actualidad los registros de la especie son numerosos, en la mayoría de las islas del archipiélago, en donde las poblaciones han ido creciendo, siendo numerosos en algunos enclaves. Ya se ha comprobado que se reproducen de una forma natural (hembras ovígeras y asentamiento de juveniles) y quizás le haya beneficiado el calentamiento del agua de mar que se está produciendo en las Islas, siendo este uno de los factores más probables de su gran éxito neocolonizador.

En general, muchas de las especies de decápodos son gregarias con altas densidades poblacionales. Esto, unido a su valor comercial como marisco, lo hace un recurso económico de gran importancia en muchos países. En el caso de $C$. ruber, entraría en esta calificación, ya que sería la «nécora canaria», ya que hasta el 
momento no existía en aguas de Canarias una especie con estas características. Por otra parte, digamos que, en relación con su nicho ecológico, no ocupa el lugar de otro cangrejo similar, al menos en lo observado en algunos enclaves de Tenerife (ver siguiente apartado para mayor detalle). Sí se ha observado que es presa de los cefalópodos, sobre todo de pulpos y sepias. En Canarias, los recursos marisqueros son muy escasos y fácilmente agotables, es por ello por lo que existen leyes de protección de todas aquellas especies vulnerables y de poblaciones mermadas. No pensamos que $C$. ruber pueda rellenar un vacío en este sentido; en todo caso, pensamos que el control de las poblaciones sería más o menos fácil, permitiendo su marisqueo, en caso de que las poblaciones vayan en aumento. Se trata de un cangrejo que incluso se puede mariscar a mano, con equipo ligero de buceo, y también se podrían utilizar nasas selectivas, de esta forma se podrían controlar las poblaciones. En Canarias, actualmente uno de los platos más populares de marisco de crustáceos son los camarones del género Plesionika, ya que como recurso de marisqueo con nasas profundas es rentable en las Islas y muy apreciado por los visitantes. Existe además precedente en España, concretamente en el delta del Ebro, donde el portúnido invasor Callinectes sapidus ya se ha comercializado como marisco (Fuentes et al., 2019). En nuestro caso, para proponer a $C$. ruber como recurso, se necesitaría hacer un muestreo más detallado de las poblaciones existentes para obtener los primeros datos demográficos y ecológicos que ayuden a estudiar su biomasa y evalúen correctamente el posible impacto en el medio natural, poniendo en marcha, si fuese necesario, métodos de capturas y zonas de prioridad para su pesca.

CRUSTÁCEOS DECÁPODOS LITORALES DE INTERÉS MARISQUERO QUE PODRÍAN COMPETIR CON CRONIUS RUBER

En el litoral costero de Canarias, los crustáceos decápodos más frecuentes representados por poblaciones relativamente numerosas son varias especies pertenecientes a la superfamilia Grapsoidea. Estas son especies bien conocidas por los mariscadores, ya sea por su consumo como marisco o como cebo o carnada, para la pesca de la «vieja». El «cangrejo blanco» (Plagusia depressa) (fig. 5a) constituye uno de los mariscos más apreciados en Canarias, por el extraordinario sabor de su carne (Barquín et al., 2005), que se podría comparar con el de la especie objeto del presente trabajo (Cronius ruber) (fig. 5b), y con otra especie costero-litoral presente en Canarias, el centollo de patas cortas (Maja brachydactyla) (fig. 5c). Descartamos en este apartado las especies de langostas, por ser grupos con otra ecología y, en general, de hábitos más profundos y crípticos que la especie en cuestión.

De las tres especies más conspicuas de grápsidos frecuentes en Canarias: P. depressa, Grapsus adscensionis (fig. 5d) y Percnon gibbesi (fig. 5e), quizás la que más entraría con solapamientos de hábitat con $C$. ruber sería el cangrejo araña $(P$. gibbesi), ya que, de los tres grápsidos, es el que tiene un comportamiento más sublitoral, y el de menor porte, ya que por su tamaño se marisquea en las Islas, siendo utilizado como cebo de carnada de "vieja», sobre todo los ejemplares juveniles que se colectan durante la bajamar. Las otras dos especies ocuparían biotopos bastante 

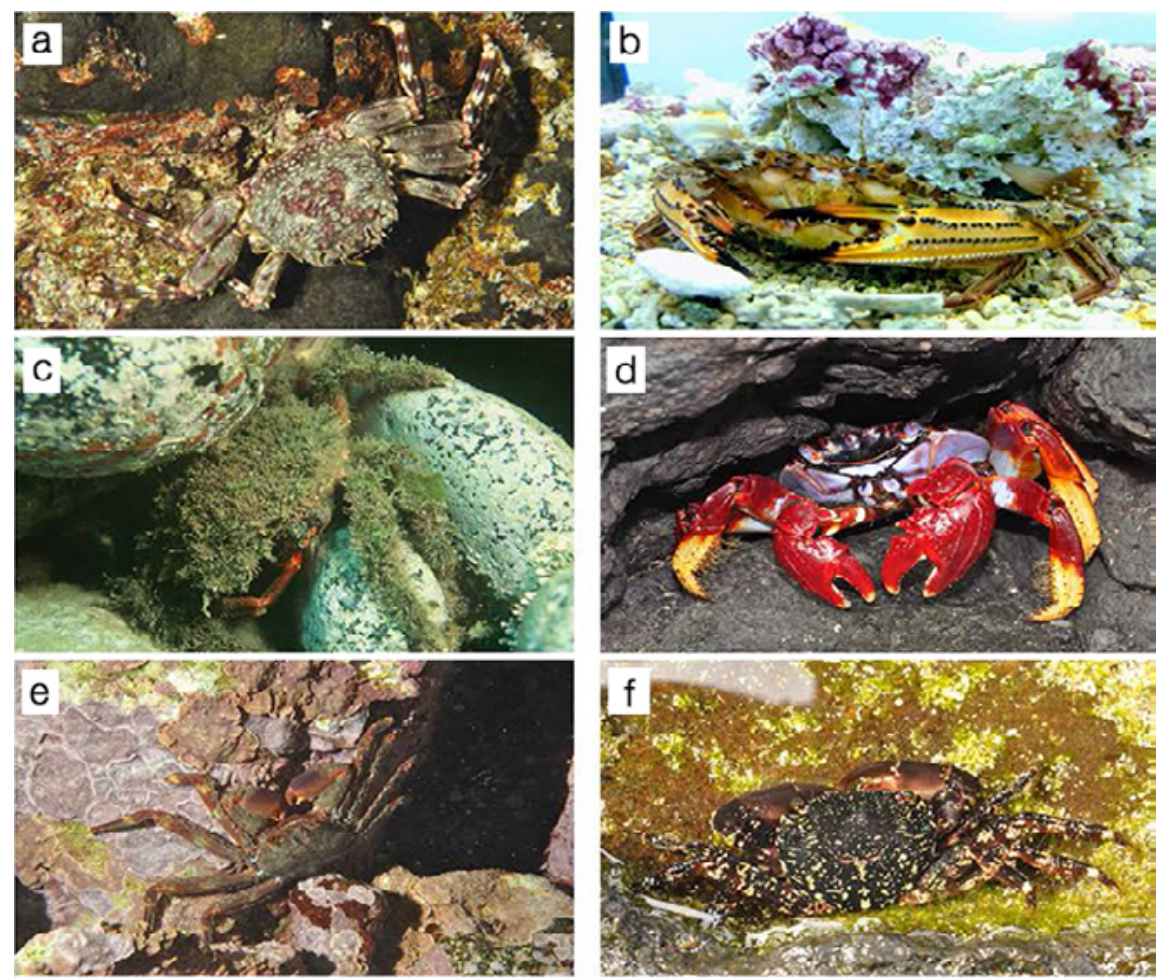

$\stackrel{\boldsymbol{m}}{\boldsymbol{m}}$
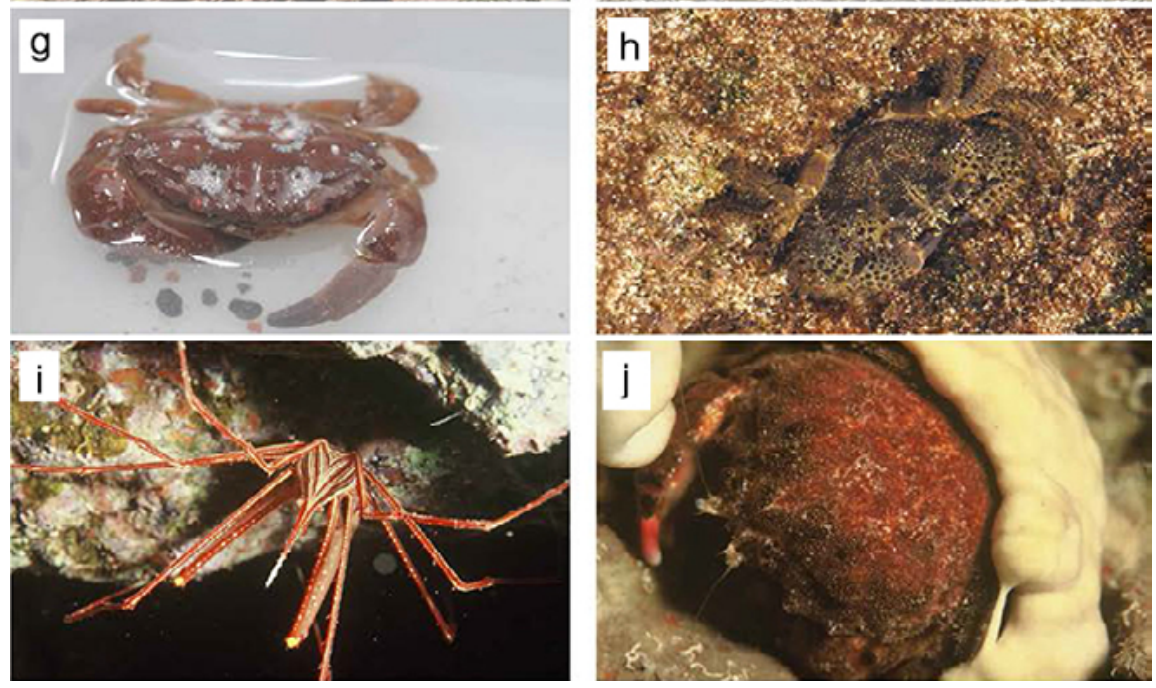

Figure 5. Crustáceos decápodos litorales de Canarias: a. Plagusia depressa (cangrejo blanco); b. Cronius ruber (nécora tropical); c. Maja brachydactyla (centollo); d. Grapsus adscensionis (cangrejo moro macho); e. Percnon gibbesi (cangrejo araña); f. Pachygrapsus marmoratus (juyón); g. Xantho poressa (carnada de viejas); h. Eriphia verrucosa (jaca); i. Stenorhynchus lanceolatus (araña de patas largas); j. Dromia personata. Fotos realizadas por José Carlos Hernández. 
diferentes a $C$. ruber, pues se localizan en los niveles más altos de marea, incluso G. adscensionis (cangrejo moro) llega hasta zonas supralitorales, siendo el cangrejo más visible en el litoral isleño, sobre todo en escolleras artificiales, ramoneando o simplemente soleándose; aunque el porte del cangrejo moro suele ser mayor que el cangrejo blanco, pues puede superar los $25 \mathrm{~cm}$; no obstante, su carne es menos apreciada como marisco, ya que es bastante amarga (Barquín et al., 2005). Otras especies de grápsidos de menor porte, abundantes en los ambientes intermareales pedregosos, son el cangrejo juyón o juyona, que se utiliza como carnada para la pesca de la vieja, entre otras especies de peces; el género Pachygrapsus (fig. 5f), con varias especies registradas en el litoral de Canarias, se suelen recolectar por volteo de piedras o callaos en el intermareal, ya que se trata de especies esciáfilas, que permanecen infralapidícolas durante las horas diurnas en la bajamar. Estas especies comentadas difícilmente competirían con C. ruber.

Además de los cangrejos juyones (Pachygrapsus), utilizados como cebo de pesca, también se recolectan otras especies presentes en el intermareal, como por ejemplo Xantho poressa (fig. 5g) y Xantho incisus, especies de la superfamilia Xanthoidea, son especies de pequeño tamaño que se recolectan también por el volteado de piedras durante la bajamar en el litoral isleño. Todas estas últimas especies, más o menos crípticas, ocupan biotopos muy diferentes al frecuentado por $C$. ruber. Existen en Canarias otras especies de cangrejos que ocupan biotopos en el intermareal rocoso, la especie de mayor tamaño es la jaca (Eriphia verrucosa) (fig. $5 \mathrm{~h}$ ), un cangrejo de quelas muy potentes que cada vez es más escaso en el litoral de Canarias; los ejemplares de mayor tamaño eran capturados como marisco, ya que se trata también de una especie de carne muy apreciada.

La superfamilia Majoidea también se encuentra bien representada con varias especies en el litoral canario, aunque se trata de especies menos visibles que las tres anteriores, ya que se mimetiza perfectamente con el fondo que la rodea; además, son más activas de noche, pues también tienen un comportamiento esciáfilo. La araña de patas largas (Stenorhynchus lanceolatus) (fig. 5i) es una especie bastante común y se puede ver desde los charcos intermareales, muy a menudo se refugia en zonas de oquedades y entradas de cuevas submarinas. Por lo que la especie que ocupa biotopos más afines a $C$. ruber es Maja brachydactyla (centollo de Canarias), especie que se encuentra en fondos de 2 a $20 \mathrm{~m}$ de profundidad, o incluso más, en fondos rocosos, aunque también pueden recorrer zonas arenosas, los ejemplares más jóvenes con camuflaje de algas sobre el caparazón y tendencia a habitar en zonas resguardadas tanto de barlovento como de sotavento. Se trata de especies muy abundantes en zonas con aguas ricas en nutrientes, como por ejemplo en las costas de Galicia, donde la centolla o centollo (Maja squinado) y la nécora (Necora puber) son especies de marisqueo en esas latitudes y de gran importancia económica. En Canarias, con aguas oligotróficas en la mayor parte de su litoral, las poblaciones son bastante escasas, en el caso de $M$. brachydactyla y una tercera especie de pequeña centolla o santorra, Maja goldziana, que se encuentra a mayor profundidad (González-Pérez, 1995), y a veces es capturada en las artes de los pescadores (nasas).

Quedarían por nombrar algunas especies de cangrejos litorales de porte medio, frecuentes en fondos someros arenosos que llegan hasta los caulerpales y 
sebadales más profundos, nos referimos a dos especies de la superfamilia Calappoidea, Calappa granulata y Cryptosoma cristatum, la primera de mayor porte y gran potencia de quelas, ambas especies son capaces de desaparecer a la vista del buceador enterrándose completamente en la arena, especies que cada vez son más escasas en los fondos arenosos insulares. Una última especie, también de ambientes someros, que incluso puede aparecer en el intermareal, es Dromia personata (fig. 5j), de la superfamilia Dromioidea, especie frecuente en tramos de entrada a cuevas submarinas y que se asocia con esponjas que utiliza como artilugio de enmascaramiento, sus poblaciones son poco numerosas y de difícil observación debido a sus hábitos esciáfilos.

\section{AGRADECIMIENTOS}

Agradecemos a Isidro González y Gabriel Dorado su inestimable ayuda y apoyo. 


\section{REFERENCIAS}

Abelló P. 1989. Feeding habits of Macropipus tuberculatus (Brachyura, Portunidae) off the Catalan coast (NW Mediterranean). Misc. Zool. 13: 45-50.

Barquín-Díez, J., Núñez-Fraga, J., Falcón-Toledo, J.M. y Brito-Hernández, A. 2005. Fauna marina, los invertebrados (pp. 325-342). En Patrimonio natural de la isla de Fuerteventura, medio físico, el mundo vegetal y la fauna. Edit. Rodríguez-Delgado, O. Centro de la Cultura Popular Canaria.

Brito, A., López, C., Ocaña, O., Herrera, R., Moro, L., Monterroso, O., Rodríguez, A., Clemente, S. y Sanchez, J.J. 2017. Colonization and expansion of potentially invasive coral species in the Canary Islands in traduced through oil platforms. Vieraea, 45:65-82.

De Grave, S., Pentcheff, N.D., Ahyong, S.T., Chan, T.-Y., Crandall, K.A., Dworschak, P.C., Felder, D.L., Feldmann, R.M., Fransen, C.H.J.M., Goulding, L.Y.D., Lemaitre, R., Low, M.E.Y., Martin, J.W., NG, P.K.L., Schweitzer, C.E., Tan, S.H., Tshudy, D. y Wetzer, R. 2009. A classification of living and fossil genera of decapod crustaceans. The Raffles Bulletin of Zoology, Suppl. 21: 1-109.

FALCón, J.M. 2015. Ictiofauna de las Islas Canarias. Análisis biogeográfico. Tesis doctoral, Universidad de La Laguna, 310 pp.

Fransen, C.H.J.M. 1991. Preliminary report on Crustacea collected in the eastern part of the North Atlantic during the CANCAP and Mauritania. Expeditions of the former Rijksmuseum van Natuur-lijke Historie, Leiden. National Natuurhistorich Museum, Leiden, 200 pp.

Fransozo, A., Mantelatto, F.L. y Nebecc, G.B. 2002. La primera etapa zoeal del género Cronius (Brachyura, Portunidae) de la costa brasileña, que eclosionó en un laboratorio. Journal of Plankton Reseacrch, 24 (11):1237-1244.

Fraschetti, S., Terlizzi, A. y Boero, F. 2008. How many habitats are there in the sea (and where)? Journal of Experimental marine biology and ecology, 366: 309-332.

Fuentes, M.A., Torrent, L., Barrera, S. y Boix, D. 2019. Rapid invasion of the American blue crab Callinectes sapidus Rathbun, 1896 in the North-East of the Iberian Peninsula. BioInvasions Records 8(1): 113-118.

GozÁlez-PÉrez, J.A. 1995. Catálogo de los crustáceos decápodos de las islas Canarias. Gambas, langostas y cangrejos. Edit. Turquesa, $282 \mathrm{pp}$.

González, J., Triay-Portella, R., Escribano, A. y Cuesta, J. 2017. Northernmost record of the pantropical portunid crab Cronius ruber in the eastern Atlantic (Canary Islands): natural range extension or human-mediated introduction? Scientia Marina, 81(1): 81-89.

Hernández, C. 2015. Sistema de carbono y productividad primaria en zonas costeras de Canarias en un contexto de Cambio Global. Tesis doctoral, Universidad de La Laguna. 210 pp.

Holthuis, L.B. 1987. «Vrais crabes», pp. 322-367. In W. Fischer, M.-L. Bauchot \& M. Schneider (ed.): Fiches FAO d'identification des espèces pour les besoins de la pêche. (Révision 1). Méditerranée et mer Noire. Zone de pêche 37. Volume I. Végétaux et Invertébrés. FAO, Rome.

Косн, M. y Ďuriš, Z. 2016. Notes on distribution of some portunid crabs in the Mediterranean Sea (Decapoda: Brachyura: Portunidae). Acta Mus. Siles. Sci. Natur. 65: 117-128.

Le Loeuff P. y Intès A. 1968. La faune benthique du plateau continental de Côte d'Ivoire, récoltes au chalut, abondance-répartition-variations saisonnières (Mars 1966-Février 1967). Centre de Recherches Océanographiques, Abidjan, Doc. Scient. Provisoire 25: 1-78. 
Manning R.B. y Holthuis L.B. 1981. West African Brachyuran Crabs (Crustacea: Decapoda). Smithson. Contr. Zool. 306: 1-379.

Rathbun, M. J. 1930. The cancroids crabs of America of the families Euryalidae, Portunidae, Atelcyclidae, cancridae, and Xanthidae. Bull.U. S. Nat. Mus., 152: 1-609.

Rodríguez, B.D. 1982. Cangrejos de la família portunidae (Decapoda, Brachyura) del Caribe colombiano. An. Inst. Inv. Mar. Punta de Betin, 12: 137-184.

Schäfer, S., Monteiro, J., Castro, N., Riloy, G. y Canning-Clode, J. 2019. Cronius ruber (Lamarck, 1818) arrives to Madeira Island: a new indication of the ongoing tropicalization of the northeastern Atlantic. Marine Biodiversity.49: 2699-2707.

SPIVAK, E.D. 1997. Los crustáceos decápodos del Atlántico sudoccidental (25ㄷ5ㅇ S): distribución y ciclos de vida. Investig. Mar., 25: 69-91.

Templado-González, J., Ballesteros-Sagarra, E., Brito-Hernández, A., Borja-Yerro, A., Galparsoro-Iza, I., Martín-García, L. y Serrano-López, A. 2012. Inventario Español de Hábitats y Especies Marinos. Guía interpretativa: inventario español de hábitats marinos. Ministerio de Agricultura, Alimentación y Medio Ambiente, Madrid, 229 pp.

Triay-Portella, R., Martín, J.A., González, J.A., Lorenzo, J.M. y Pajuelo, J.G. 2018. Conocer al invasor: dispersión del decápodo no nativo Cronius ruber en los ecosistemas marinos de Gran Canaria (Islas Canarias) VI International Symposium on Marine Sciences Vigo (Spain).

Williams, A.B. 1965. Marine decapods crustaceans of the Carolinas. Fishery Bulletin, 65 (1): 1-293. 